EXTENDED REPORT

\title{
Corneal sensation after myopic and hyperopic LASIK: clinical and confocal microscopic study
}

\author{
M A Bragheeth, H S Dua
}

Br J Ophthalmol 2005;89:580-585. doi: 10.1136/bjo.2004.046888

See end of article for authors' affiliations

.....................

Correspondence to: Professor Harminder $\mathrm{S}$ Dua, Division of Ophthalmology and Visual Sciences, B floor, Eye Ear Nose Throat Centre, University Hospital, Queens Medical Centre, Nottingham NG7 2UH, UK; harminder.dua@ nottingham.ac.uk

Accepted for publication 19 May 2004

\begin{abstract}
Aim: To assess the long term (1 year) effect of myopic and hyperopic LASIK on corneal sensation and innervation.

Methods: 83 eyes of 43 patients having LASIK were evaluated. According to the preoperative spherical equivalent, the eyes were divided into three groups: group 1, myopia from -0.75 to $-6.00 \mathrm{D}$; group 2, myopia from -6.25 to $-11.50 \mathrm{D}$; and group 3, hyperopia from 1.25 to $5.00 \mathrm{D}$. Corneal sensation was measured and in vivo confocal microscopy (IVCM) was done at the central cornea before, and at 1 month, 3 months, 6 months, and 1 year after LASIK.

Results: The mean corneal sensation in group 1 was greater than in groups 2 and 3 at all postoperative measurements. The difference between group 1 on one hand and groups 2 and 3 on the other hand was statistically significant at 1 month and 3 months after LASIK and was not statistically significant afterwards. IVCM study of 27 eyes revealed that the number and length of nerve fibre bundles in the sub-basal region decreased after LASIK and was significantly lower at all times after surgery despite the return of corneal sensation to preoperative level.

Conclusion: After LASIK, central corneal sensitivity is decreased for as long as 6 months or more. The results suggest that lamellar cutting of the cornea during LASIK impairs corneal sensitivity and is related to the ablation depth. The diameter of ablation too may contribute to this drop in sensitivity. The return of corneal sensations does not directly correlate with the regeneration of nerve fibres as determined by confocal imaging. Sensations return to normal values before complete restoration of normal innervation if this indeed ever occurs.
\end{abstract}

$\mathrm{T}$ e sensory innervation of the cornea is derived from the ophthalmic and maxillary branches of the trigeminal nerve. These nerve fibres are myelinated until they penetrate the limbus and form thick nerve bundles surrounded by the Schwann cells in the anterior third of the stroma. ${ }^{1}$ In human cornea these bundles divide dichotomously or trichotomously, bend 90 degrees, lose their Schwann cell sheath, and penetrate Bowman's layer. ${ }^{23}$ Then they bend again almost at right angles forming the basal epithelial/subepithelial nerve plexus between the basal epithelial cells and Bowman's layer. Fibres of this plexus branch both horizontally and vertically sending nerve terminals between the epithelial cells. ${ }^{4}$ There are three classes of these corneal sensory receptors-mechanoreceptors, polymodal receptors, and thermoreceptors-that are responsible for mechanical, chemical, and thermal sensation, respectively. A- $\delta$ fibres react to mechanical sensation and the $\mathrm{C}$ fibres to temperature. ${ }^{5}$ Some of the stromal keratocytes are innervated by stromal nerve fibres and both basal and wing cells have been supposed to be directly innervated by epithelial nerve fibres. ${ }^{3}$

Normal corneal sensitivity is essential to normal corneal structure and function. Touching the cornea triggers one of the most sensitive protective reflexes of the human body. The threshold of sensitivity, especially in the centre of the cornea, is exceedingly low, so pathological changes can be diagnosed early and precisely and corneal sensation can be used for diagnosis, follow up, and even for prognosis of various corneal disorders.

Loss of normal corneal sensation may compromise the protective blink reflex, delay epithelial wound healing, decrease tear flow, and be associated with neurotrophic keratitis, sterile corneal melts, and infectious keratitis. ${ }^{6}$
Cochet and Bonnet ${ }^{7}$ have shown that sensitivity of the cornea is greatest at its centre and decreases towards the periphery. The upper part of the cornea is the least sensitive, and the temporal part is highly sensitive.

During laser in situ keratomileusis (LASIK) surgery the superficial stromal nerves are cut in the flap margin and the nerves in the stromal bed under the flap are subsequently exposed to excimer laser photoablation. The LASIK flap is usually $160 \mu \mathrm{m}$ thick and the additional ablation depth of the excimer laser varies according to the desired myopic correction. Both factors (flap cutting and laser ablation) contribute to the innervation damage, which develops following LASIK surgery.

We undertook a prospective study to assess the effect of myopic and hyperopic LASIK on corneal sensation and corneal innervation.

\section{PATIENTS AND METHODS}

In a prospective, comparative, non-randomised consecutive case study, 83 eyes of 43 patients, 11 male and 32 female, with a mean age of 31.2 years (range 23-40 years) underwent LASIK. Surgeries were performed by the same surgeon (HSD) from August 2001 to December 2002. According to the preoperative spherical equivalent, the eyes were divided into three groups: group 1, myopia from -0.75 to $-6.00 \mathrm{D}$; group 2, myopia from -6.25 to $-11.50 \mathrm{D}$; and group 3, hyperopia from 1.25 to $5.00 \mathrm{D}$ (table 1). All eyes had normal corneal sensation before LASIK (see table 2).

Abbreviations: BSCVA, best spectacle corrected visual acuity; IVCM, in vivo confocal microscopy; LASIK, laser in situ keratomileusis; NFBs, nerve fibre bundles; UCVA, uncorrected visual acuity 


\begin{tabular}{lll}
$\begin{array}{l}\text { Table 1 } \\
\text { groups }\end{array}$ & Classification of patients into the different \\
\hline Group & Spherical equivalent (D) & No of eyes \\
\hline Group 1 & -0.75 to -6.00 & 46 \\
Group 2 & -6.25 to -11.50 & 27 \\
Group 3 & +1.25 to +5.00 & 10 \\
\hline
\end{tabular}

Preoperative examinations included personal medical ocular history, uncorrected visual acuity (UCVA), best spectacle corrected visual acuity (BSCVA), corneal topography (with the Eye Sys system 2000, Eye Sys Technologies, Houston, TX, USA), ultrasonic pachymetry (Advent Pachymeter; Mentor), manifest refraction, tonometry, slit lamp microscopy, corneal sensitivity testing (using the Cochet-Bonnet aesthesiometer; Luneau, Paris, France), and dilated fundus examination. Pupil size was measured with the Infrared Pupilscan II (Keeler, Broomall, PA, USA) in a mesopic room environment. In vivo confocal microscopy (IVCM) was done for 27 eyes ( 10 eyes from group 1, 10 eyes from group 2, and seven eyes from group 3). Patients recruited for IVCM were age and sex matched. Informed consent was obtained from all patients after they received a detailed description of the surgical procedure and its known risks, and if they wore contact lenses they had been advised to discontinue soft lens wear for 2 weeks or hard contact lens wear for 1 month.

The standard LASIK technique was used for all patients. Treatment was done under topical anaesthesia using amethocaine preservative-free hydrochloride (Minims; Chauvin, Romford, UK) eye drops twice; first just before introduction of the patient to the operating room and a second drop of topical anaesthetic after application of the eye speculum.

The automated microkeratome (Hansatome) was used to cut a superiorly hinged flap of $160 \mu \mathrm{m}$ thickness for all cases. The flap intended diameter was $8.5 \mathrm{~mm}$ for myopic cases and $9.5 \mathrm{~mm}$ for hyperopic cases.

The laser ablation, in our patient series, was performed using the VISX Star 3 excimer laser with the following parameters: wavelength $193 \mathrm{~nm}$, fluence $160 \mathrm{~mJ} / \mathrm{cm}^{2}$, repetition rate $10 \mathrm{~Hz}$, ablation zone diameters between $6 \mathrm{~mm}$ and $6.5 \mathrm{~mm}$, transition zone $0.35 \mathrm{~mm}$.

The desired treatment parameters were entered into the laser's computer and laser ablation was then performed in the stromal bed. The target postoperative refraction was emmetropia in all eyes.

At the end of the operation, a protective transparent shield was placed over the eye and secured with surgical tape. No patching was used. Patients were examined 30 minutes following the procedure to assure proper flap position. Chloramphenicol $0.5 \%$ eye drops four times daily for 1 week and prednisolone acetate $0.5 \%$ eye drops four times daily for 2 weeks, were prescribed to be used after surgery. The following day, the shield was removed, and the eye was examined. Unpreserved artificial tears were recommended for mild irritation caused by dry eye in some cases.

Postoperative examinations at 1, 3, 6, and 12 months after LASIK included UCVA, BSCVA, manifest refraction, ultrasonic pachymetry, corneal topography, corneal sensitivity testing, and complete slit lamp biomicroscopic evaluation of the anterior and posterior segments.

Using the Cochet-Bonnet aesthesiometer (CBA) (Luneau, Paris, France), central corneal sensitivity was measured before and at 1, 3, and 6 months, and 1 year after surgery. Normal levels of corneal sensitivity were considered $>50 \mathrm{~mm}$ for the central cornea. Full recovery was defined as recovery to the preoperative level of corneal sensitivity. Measurement of the central corneal sensation was done using the technique described by Matsui et al $^{8}$ and Kumano et al. ${ }^{9}$ The instrument consists of a nylon filament $60.0 \mathrm{~mm}$ long and $0.12 \mathrm{~mm}$ in diameter. The force exerted by the filament when it touches the cornea is inversely proportional to its length. All measurements were taken by the same observer and in the evenings (between $6 \mathrm{pm}$ and $8 \mathrm{pm}$ ). The CBA has limited sensitivity as a method of measuring corneal sensations but is the most practical one available.

Sensitivity measurement began at the maximal filament length, $60.0 \mathrm{~mm}$, which corresponds to the lowest possible pressure. A bend in the nylon filament provides an objective measurement of contact. If the patient did not feel the filament at $60.0 \mathrm{~mm}$, the filament length was reduced by $5.0 \mathrm{~mm}$ each time before retesting. Retesting was continued until the patient was able to feel the nylon thread, which constitutes a positive response. The filament was maintained perpendicular to the cornea at all times. Measurements were recorded as millimetres of Cochet-Bonnet filament length. IVCM was performed in 27 eyes at 1, 3, and 6 months and at 1 year after LASIK treatment.

Central corneas were scanned throughout their full thickness by white light, tandem, slit scanning confocal microscopy (Confoscan P4, Tomey) before and at 1, 3, 6, and 12 months after LASIK.

The microscope objective lens was cleaned with 70\% isopropyl alcohol wipes before and after each examination. The corneal surface was anaesthetised using 1\% amethocaine hydrochloride eye drops (Minims; Chauvin, Romford, UK). A drop of $2.5 \%$ hydroxypropyl methylcellulose (Goniosol; Ciba Vision) was placed on the tip of the objective lens as an optical coupling medium, and the lens was manually advanced until the medium contacted the surface of the central cornea. A full thickness scan, consisting of a series of confocal images, was recorded as the focal plane was advanced from anterior to the epithelium to posterior to the endothelium. Real time images were captured using a low light level video camera and the images were recorded on an S-VHS videotape (Fuji Magnetics, Kleve, Germany) using a video cassette recorder. Digital images were stored on a computer workstation (PC Pentium, Tomey Base, Confo Scan P4, Tomey) at 25 frames/s. Each image represented a coronal section approximately $475 \times 350 \mu \mathrm{m}$ (horizontal $\times$ vertical) and was separated from adjacent images by approximately $2.6 \mu \mathrm{m}$. On each visit, the cornea was scanned through its full thickness two to four times. All scans were within the central $4 \mathrm{~mm}$ of the cornea representing full thickness samples of the central cornea. Clear digital images were selected by the same investigator, for all patients for all visits, for further analysis.

Nerve fibre bundles (NFBs) appeared as bright, well defined, linear structures that were sometimes branched and usually appeared in several consecutive frames. All confocal scan images of sufficient quality for sub-basal nerves visualisation were evaluated for each patient. The NFBs per scan in two to four scans per eye per visit was determined and measured in the sub-basal region and in the stromal flap (layer between the most anterior keratocyte and the flap interface). Based on the confocal images, the subbasal NFBs were grouped into four different categories based on their morphology ${ }^{10}$ : (1) no nerve images, (2) only short $(<200 \mu \mathrm{m})$ NFBs, (3) long $(\geqslant 200 \mu \mathrm{m})$ NFBs without interconnections, and (4) long NFBs with interconnections (see fig 2).

Statistical analysis was performed using Excel (Microsoft, Inc) and SPSS for Windows vll.0.1. Results are presented as means (SD) and were compared with the Student's two tailed $t$ test for unpaired samples. Mann Whitney test, a 
Table 2 Mean corneal sensation (SD) before and after LASIK shown as millimetres of Cochet-Bonnet filament length at each time point for each of the three groups examined

\begin{tabular}{|c|c|c|c|c|}
\hline Time & Group 1 & Group 2 & Group 3 & p Value \\
\hline Preoperative $^{*}$ & $57.93(04.47) \mathrm{mm}$ & $56.25(10.62) \mathrm{mm}$ & $58.00(02.58) \mathrm{mm}$ & 0.43 \\
\hline 1 month postop & $39.86(10.17) \mathrm{mm}$ & $29.50(17.24) \mathrm{mm}$ & $29.00(14.87) \mathrm{mm}$ & 0.01 \\
\hline 3 months postop & $45.71(08.99) \mathrm{mm}$ & $37.69(13.17) \mathrm{mm}$ & $36.00(11.25) \mathrm{mm}$ & 0.02 \\
\hline 6 months postop & $48.01(09.76) \mathrm{mm}$ & $47.08(11.75) \mathrm{mm}$ & $45.65(07.75) \mathrm{mm}$ & 0.09 \\
\hline 1 year postop & $52.25(06.17) \mathrm{mm}$ & $51.00(07.07) \mathrm{mm}$ & $51.87(08.84) \mathrm{mm}$ & 0.16 \\
\hline
\end{tabular}

$* 8,6$, and 4 eyes in groups 1,2 , and 3 respectively wore contact lenses preoperatively. The average corneal sensation as measured by Cochet-Bonnet aesthesiometer for these patients was $56.87 \mathrm{~mm}, 55.83 \mathrm{~mm}$, and $58.75 \mathrm{~mm}$. There was no statistical difference in preoperative corneal sensation between contact lens wearers and that of the rest of the group.

non-parametric test, was used to analyse the data that are not normally distributed. Median numbers of long sub-basal NFBs were related to corneal sensation in each group at each follow up visit by using the Friedman test, a non-parametric version of the repeated measures analysis of variance. A p value of less than 0.05 was considered statistically significant.

\section{RESULTS}

Mean preoperative and postoperative central corneal sensations of all groups are shown in table 2 . and in figure 1 .

The preoperative mean corneal sensation was 57.93 (4.47) $\mathrm{mm}, 56.25(10.62) \mathrm{mm}$, and $58.00(2.58) \mathrm{mm}$ at the central cornea in groups 1, 2, and 3 respectively. These baseline preoperative corneal sensation differences between the three groups were not statistically significant $(\mathrm{p}>0.05)$.

One month after LASIK, the mean corneal sensation in group 1 (39.86 (10.17) $\mathrm{mm}$ ) was greater than in groups 2 and 3 (29.50 (17.24) $\mathrm{mm}$ and 29.00 (14.87) mm, respectively). The difference between group 1 on one hand and groups 2 and 3 on the other hand was statistically significant $(\mathrm{p}<0.01)$.

Three months after LASIK, the mean corneal sensation in group 1 (45.71 $(08.99) \mathrm{mm})$ was greater than the other groups 2 and 3 (37.69 (13.17) $\mathrm{mm}$ and 36.00 (11.25) mm, respectively). The difference was statistically significant $(\mathrm{p}=0.01)$.

The difference between the corneal sensation, at the 6 month follow up, in group 1 (48.91 (09.76) mm); group 2 (47.08 (11.57) mm); and group 3 (45.65 (07.75) $\mathrm{mm}$ ) was not statistically significant $(\mathrm{p}>0.05)$.

One year after LASIK treatment, the difference between the mean corneal sensation in group $1(52.25(06.17) \mathrm{mm})$, group 2 (51.00 (07.07) $\mathrm{mm})$, and group $3(51.87(08.84) \mathrm{mm})$ was not statistically significant $(\mathrm{p}>0.05)$. The difference between the mean preoperative corneal sensation and the

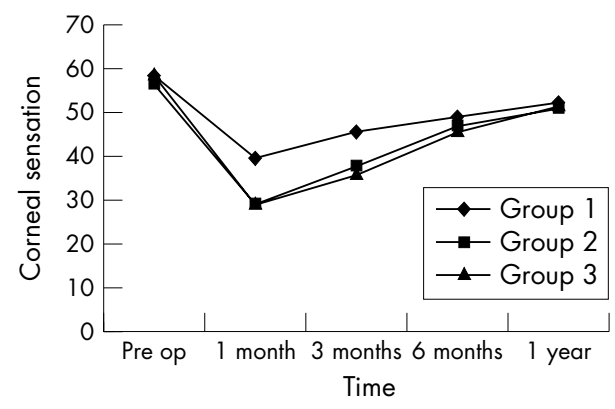

Figure 1 Graph showing the mean corneal sensitivity as measured in millimetres with the Cochet-Bonnet aesthesiometer (y axis) over the follow up period of 1 year (x axis). Group 1 (low myopes) shows a significant difference compared to the other two groups (high myopes and hyperopes). By 1 year all three groups were similar but had not recovered to preoperative levels. mean corneal sensation 1 year after LASIK treatment was not statistically significant in the three groups $(\mathrm{p}>0.05)$.

Slit lamp biomicroscopy and fluorescein staining revealed evidence of superficial punctate keratitis (SPK) in 13 (15.6\%) eyes at 1 month post-LASIK (four from group 1, six from group 2, and three from group 3). At 3 months post-LASIK, the number of eyes that had SPK were eight eyes (9.6\%) (two, four, and two in the three groups, respectively) and at 6 months post-LASIK only four eyes $(4.8 \%)$ showed SPK (one, two, and one in the three groups respectively).

IVCM study of 27 eyes, before and after LASIK, revealed that the number and length of NFBs in the sub-basal region decreased after LASIK (table 3 ) and was significantly lower at all times after surgery than it was before surgery $(p<0.001)$. It increased 6 and 12 months after LASIK, but remained less than the preoperative value. The number and length of the sub-basal NFBs (fig 2), was lower in groups 2 and 3 (high myopia and hyperopia groups) than in group 1 (mild to moderate myopia).

In the flap stroma, the stromal NFBs appeared as thick lines that could be seen through several successive optical sections. The number and length of NFBs after surgery were also less than before LASIK but we could not quantify them because of their small number and inconsistency of their detection. We could not also study them statistically for the possible difference and correlation to the LASIK treatment.

\section{DISCUSSION}

The cornea is the most richly innervated surface tissue of the body. Interest in the sensitivity of the cornea dates back to the 19th century. The German physiologist Von Frey in 1894, concluded that pain was the only sensation perceived by the cornea.

Before describing the changes in corneal sensation after LASIK, it is important to be aware of the following normal physiological variations in corneal sensitivity. Corneal sensitivity varies with eccentricity; the cornea is most sensitive at its centre and sensitivity decreases gradually towards the periphery. This shows a good correlation with the distribution of the sensory nerve terminals. A diurnal variation in corneal sensitivity has been noted; the sensitivity is highest in the evening and lowest in the morning, a trend that seems to be related to corneal hypoxia during sleep. Diurnal variation is an important consideration in longitudinal studies where repeated measurements are taken over time. Corneal sensitivity appears to vary little over the first four decades of life but later it shows a gradual decline (hypoaesthesia). Sex differences also require consideration as the hormonal changes which occur during the menstrual cycle and pregnancy may be associated with changes in corneal sensitivity. ${ }^{11}$

During LASIK surgery the superficial stromal nerves are cut in the flap margin and the nerves in the stromal bed under the flap are subsequently exposed to excimer laser photoablation. 

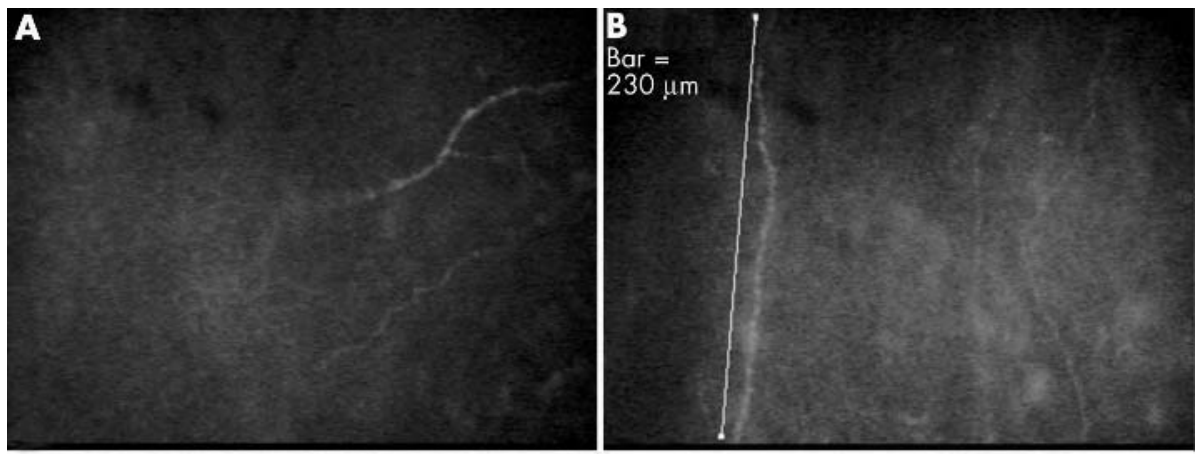

Figure 2 In vivo confocal microscopic images of the corneal nerves regenerating after LASIK. (A) Only short $(<200 \mu \mathrm{m})$ unconnected nerve fibre bundles are seen in the sub-basal area. (B) Long ( $>200 \mu \mathrm{m})$ nerve fibre bundles without interconnections are seen in the sub-basal area (bar $=230 \mu \mathrm{m})$. (C) Long nerve fibre bundles with interconnections are seen in the sub-basal area. (D) Thicker stromal nerve fibre bundles.
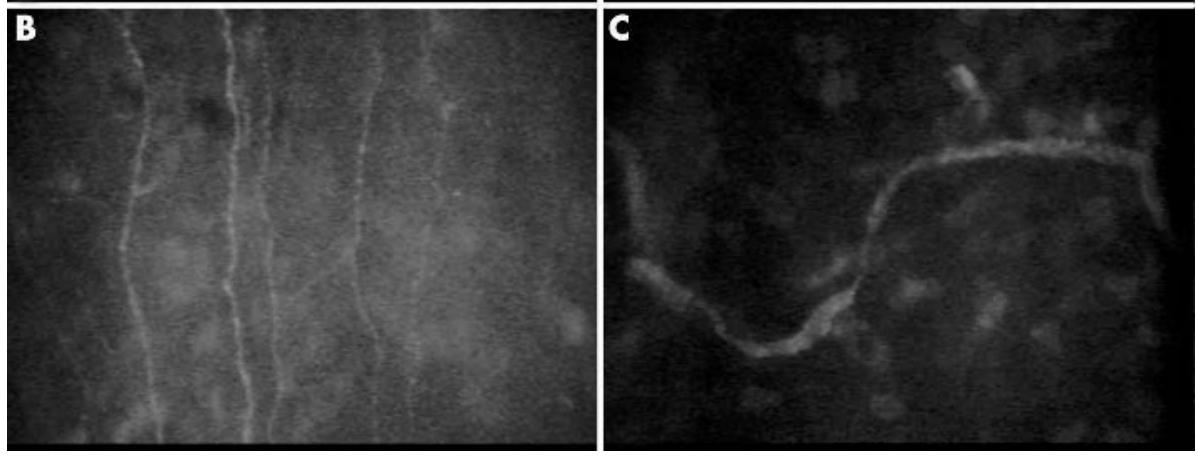

The nerves distal to a surgically induced corneal wound degenerate, thereafter the proximal trunks begin to send regenerating nerve fibres into the wounded area. ${ }^{12}$

A potentially important feature of LASIK is that it spares the epithelium, the Bowman layer, a considerable proportion of the anterior stromal nerve plexus and the corneal nerves in the hinge region, which remain unaffected. Furthermore, the flap contains an undisturbed Bowman's layer and the original Schwann cell pathways, which might facilitate the process of nerve recovery.

Aesthesiometry is a reproducibly accurate measure of corneal sensation. The most popular device for this purpose is the Cochet-Bonnet aesthesiometer (Luneau), which consists of a calibrated nylon filament for mechanical stimulation. ${ }^{13}$ It has limitations in its sensitivity as a test but is the most practical method available. The conclusions of this study should be interpreted within the limits of this method of evaluation.

Corneal sensation returned to near preoperative levels by 3 months after LASIK in group 1 (low to moderate myopia) and by 6 months in the high myopia and hyperopia groups (groups 2 and 3 respectively in our series). This level of return of corneal sensation is probably a useful level of recovery to allow corneal protection by the sensation induced blink and tearing reflexes. This observed time is substantially shorter than the previously reported time of 9.3 months $^{14}$ and longer than reported by Chuck et al, ${ }^{13}$ who observed that corneal sensation returned to near preoperative levels by 3 weeks after LASIK.

Kim and co-authors, ${ }^{15}$ reported that corneal sensitivity did not recover to the preoperative level by 6 months after LASIK

Table 3 Morphological status of the sub-basal nerve fibre bundles (NFBs) at each time point for the three groups examined

\begin{tabular}{|c|c|c|c|c|}
\hline Groups & $\begin{array}{l}\text { No NFB } \\
\text { images }\end{array}$ & $\begin{array}{l}\text { Short, } \\
\text { unconnected } \\
\text { NFBs }\end{array}$ & $\begin{array}{l}\text { Long NFBs } \\
\text { without } \\
\text { interconnections }\end{array}$ & $\begin{array}{l}\text { Long NFBs with } \\
\text { interconnections }\end{array}$ \\
\hline \multicolumn{5}{|l|}{ Group 1} \\
\hline Preoperative & 0 & 0 & 1 & 9 \\
\hline 1 month after LASIK & 8 & 1 & 1 & 0 \\
\hline 3 months after LASIK & 4 & 4 & 2 & 0 \\
\hline 6 months after LASIK & 0 & 1 & 6 & 3 \\
\hline 1 year after LASIK & 0 & 0 & 4 & 6 \\
\hline \multicolumn{5}{|l|}{ Group 2} \\
\hline Preoperative & 0 & 0 & 2 & 8 \\
\hline 1 month after LASIK & 9 & 1 & 0 & 0 \\
\hline 3 months after LASIK & 5 & 4 & 1 & 0 \\
\hline 6 months after LASIK & 2 & 3 & 5 & 0 \\
\hline 1 year after LASIK & 0 & 2 & 5 & 3 \\
\hline \multicolumn{5}{|l|}{ Group 3} \\
\hline Preoperative & 0 & 0 & 1 & 6 \\
\hline 1 month after LASIK & 5 & 2 & 0 & 0 \\
\hline 3 months after LASIK & 2 & 4 & 1 & 0 \\
\hline 6 months after LASIK & 1 & 2 & 3 & 1 \\
\hline 1 year after LASIK & 0 & 0 & 4 & 3 \\
\hline
\end{tabular}


except at the hinge of the corneal flap and that the pattern of sensitivity recovery was similar among the various points.

The difference between authors in the time of corneal sensation recovery may be related to two factors: the first is the difference between the groups studied. We found a difference in the corneal sensation recovery time between low to moderate myopia, high myopia, and hyperopia. The second factor is the difference between the authors in the definition of the corneal sensation recovery. Complete corneal sensation recovery, definitely, will take longer than what we call near normal or useful corneal sensation recovery.

At 1 month post-LASIK treatment, corneal sensation in group 2 (the high myopia group) was markedly lower than in group 1 (mild to moderate myopia) and it took 6 months for the corneal sensation to recover to near normal level in group 2 compared to 3 months in group 1. The corneal sensation at one month postoperatively was significantly lower in all eyes than the preoperative level.

One of the reasons for this difference of postoperative corneal sensation between the shallow and deep ablation groups may be the correlation between depth of ablation and nerve damage. The deeper the ablation, the larger the amount of tissue removed from the stroma and the deeper the damage to nerve trunks. Therefore, it is no surprise that the groups with deeper ablation showed a greater decrease in sensation and a longer time for full recovery than did the groups with shallow ablation.

Sub-basal nerve fibre bundles (fig 2A, B, and C), composed of several axons surrounded by a Schwann cell sheath ${ }^{3}$ were easy to identify by confocal microscopy. Consequently, they have become the main objects for evaluation of corneal nerve density by IVCM ${ }^{16}{ }^{17}$ and they also serve as a useful landmark between the epithelium and Bowman's layer. They usually appear as bright, well defined, linear structures. They are sometimes branched, and they usually appear in several consecutive frames but individual nerve fibres cannot be seen with the clinical confocal microscope. Thick stromal nerve bundles (fig 2D) and their side branches, seen as reflecting lines, can easily be imaged, but quantification is difficult because their numbers are small and the same bundles travel through several consecutive $7-10 \mathrm{~mm}$ thick optical sections. ${ }^{18}$

Chiou and co-authors ${ }^{19}$ identified many linear features by clinical confocal microscopy including vessels, lattice dystrophy, posterior polymorphous dystrophy, and fungal keratitis. However, the only linear structures in normal corneas consisted of nerves. They were well delineated and had homogeneous hyper-reflectivity.

Examination of the regeneration of the subepithelial nerve plexuses after LASIK showed that, firstly, regenerating fibres appeared as short sub-basal leaches. Then by 3 months they were elongated, but interconnections were not observed before the sixth postoperative month.

Correlation between regeneration of sub-basal nerve morphology, as observed by IVCM, and corneal sensitivity, measured by Cochet-Bonnet aesthesiometer, suggested that corneas with no nerve images or short, unconnected nerve fibres, in the sub-basal layer, are associated with lower sensitivities than corneas with long nerve fibres with or without visible interconnections $(\mathrm{p}<0.001)$. That agrees with IVCM study of myopic LASIK treated eyes by Linna and coauthors. ${ }^{10}$

Like other studies, ${ }^{20}$ we also noted that re-innervation commenced from the margins of the ablation zone toward the centre. That finding explains the greater decrease in sensation in the hyperopia (group 3) and the longer time for full recovery than the mild to moderate myopia (group 1).

Disturbance of the sensory reflex arch between the cornea and lacrimal system may have accounted for the fact that a number of patients displayed dry eye symptoms and signs after LASIK. Features typical for dry eye, such as lack of the water component and impaired tear fluid stability, have been described. ${ }^{21}$ Siganos et $a l^{22}$ found that the tear secretion following LASIK was decreased during 3 months after surgery and was normalised by 6 months.

Wilson $^{23}$ reported significant punctate epithelial erosions and rose Bengal staining on the flap in $4 \%$ of patients at $1-$ 3 months after LASIK. He hypothesised that corneal epithelial rose Bengal staining that develops after LASIK is most likely attributable to LASIK induced (presumed) neurotrophic epitheliopathy, similar to that which is noted in eyes with trigeminal nerve defects caused, for example, by trauma or tumours. In our study we noted that the overall incidence of punctate keratitis was relatively low $(15.6 \%)$ at 1 month and declined with time, presumably related to recovery of corneal sensations.

Ocular surface disease related to tear fluid abnormalities and/or neurotropic phenomena represent the most common adverse effect of LASIK. ${ }^{24}{ }^{25}$ In this respect IVCM can be used to assess postoperative corneas, which show neural damage. IVCM can also be used to differentiate them from eyes with decreased tear secretion, but well regenerated innervation. Moilanen et $a^{26}$ reported a patient with a very well regenerated sub-basal nerve plexus in the central cornea but still showed subjective dry eye 5 years after PRK.

It is likely that there is a difference between patients in the sensitivity of the corneal epithelium to denervation, with some patients having symptoms of dryness without signs develop, others having symptoms and signs develop, and most having neither symptoms nor signs of epitheliopathy. Individuals with borderline dry eye are known to have an increase in symptoms for variable periods after LASIK treatment and normal individuals may start experiencing symptoms for the first time following LASIK.

Our results agree with the longitudinal studies done by Lee et $a l^{27}$ using the confocal microscopy, which suggest that morphological recovery of corneal innervation may take longer than 12 months after LASIK. In our series, confocal microscopy of the cornea 1 year after LASIK showed that sub-basal NFB did not recover to the preoperative condition even in some patients who had recovered normal corneal sensation. Probably, the number and length of sub-basal NFBs needed for normal corneal sensation are less than the average normal sub-basal NFB density or mechanical corneal sensation measurement is not very accurate.

\section{CONCLUSION}

After LASIK, corneal sensitivity is decreased in the central cornea for as long as 6 months or more. The results suggest that lamellar cutting of the cornea during LASIK impairs corneal sensitivity and that the depth of the corneal ablation affects the extent of loss of corneal sensitivity and recovery. The diameter of the flap and the ablation area may also have an effect on the degree of corneal sensation loss and time for its recovery. The deeper the ablation and the larger the flap and ablation diameter, the greater the reduction in corneal sensitivity and the longer the time for full recovery. In vivo confocal microscopy revealed LASIK induced alterations of nerve morphology in the sub-basal layer and thus enabled studying the correlation between corneal sensory innervation and sensitivity. A direct positive correlation was found between corneal sub-basal nerve bundle regeneration and corneal sensation recovery. However, as shown in this study, full recovery of the sub-basal NFBs was not achieved by the time of near normal, or useful corneal sensation recovery ( 6 months after LASIK). Lee $e t l^{27}$ found that at 1 year postLASIK, the number of sub-basal NFB remained less than half of that before LASIK. Similarly, Muller et $^{1 l^{18}}$ stated that near 
normal recovery of sub-basal NFB can be expected 2 years after LASIK.

\section{Authors' affiliations}

M A Bragheeth, H S Dua, Division of Ophthalmology and Visual Sciences, University of Nottingham, Nottingham, UK

Proprietary interest: Both authors have no propriety interest in any equipment, method, or technique used or referenced in the manuscript.

\section{REFERENCES}

1 Zander E, Weddell G. Observations on the innervation of the cornea. J Anat 1951;85:68-99.

2 Schimmelpfennig B. Nerve structures in human central corneal epithelium. Graefes Arch Clin Exp Ophthalmol 1982;218:14-20.

3 Muller L, Pels L, Vrensen GF. Ultrastructural organization of human corneal nerves. Invest Ophthalmol Vis Sci 1996:37:476-88.

4 Muller $\mathrm{U}$, Vrensen GF, Pels L, et al. Architecture of human corneal nerves. Invest Ophthalmol Vis Sci 1997;38:985-94.

5 Belmonte C, Garcia-Hirschfeld J, Gallar J. Neurobiology of ocular pain. Prog Retinal Eye Res 1997; 16:117-56.

6 Martin X. Safran A. Corneal hypoesthesia. Surv Ophthalmol 1988;33:28-40.

7 Cochet P, Bonnet R. L'ésthésie cornéenne. Sa mesure clinique. Ses variations physiologiques et pathologiques. Clin Ophtalmol 1960;4:3-27.

8 Matsui H, Kumano Y, Zushi I, et al. Corneal sensation after correction of myopia by photorefractive keratectomy and laser in situ keratomileusis. J Cataract Refract Surg 2001;27:370-3.

9 Matsui H, Kumano Y, Zushi I, et al. Recovery of corneal sensation after myopic correction by laser in situ keratomileusis with a nasal or superior hinge. $J$ Cataract Refract Surg 2003;29:757-61.

10 Linna TU, Vesaluoma MH, Perez-Santonja JJ, et al. Effect of myopic LASIK on corneal sensitivity and morphology of subbasal nerves. Invest Ophthalmol Vis Sci 2000;41:393-7.

11 Lawrenson JG, Corbett MC, O'Brart DP, et al. Effect of beam variables on corneal sensitivity after excimer laser photorefractive keratectomy. Br J Ophthalmol 1997;81:686-90.
12 Leeuw AM, Chan KY. Corneal nerve regeneration: Correlation between morphology and restoration of sensitivity. Invest Ophthalmol Vis Sci 1989;30:1980-90.

13 Chuck RS, Quiros PA, Perez AC, et al. Corneal sensation after laser in situ keratomileusis. J Cataract Refract Surg 2000;26:337-9.

14 Kanellopoulos AJ, Pallikasis IG, Donnenfeld ED, et al. Comparison of corneal sensation following photorefractive keratectomy and laser in situ keratomileusis. J Cataract Refract Surg 1997;23:34-8.

15 Kim WS, Kim JS. Change in corneal sensitivity following laser in situ keratomileusis. J Cataract Refract Surg 1999;25:368-73.

16 Linna T, Tervo T. Real-time confocal microscopic observations on human corneal nerves and wound healing after excimer laser photorefractive keratectomy. Curr Eye Res 1997;16:640-9.

17 Linna TU, Pérez-Santonja JJ, Tervo KM, et al. Recovery of corneal nerve morphology following laser in situ keratomileusis. Exp Eye Res 1998;66:755-63.

18 Muller LJ, Marfurt CF, Kruse F, et al. Corneal nerves: structure, contents and function. Exp Eye Res 2003;76:521-42.

19 Chiou AG-Y, Kaufman SC, Beuerman RW, et al. Differential diagnosis of linear corneal images on confocal microscopy. Cornea 1999;18:63-6.

20 Perez-Gomez I, Efron N. Change to corneal morphology after refractive surgery (myopic laser in situ keratomileusis) as viewed with a confocal microscope. Optom Vis Sci 2003;80:690-7.

21 Albietz JM, Lenton LM, McLennan SG. Effect of laser in situ keratomileusis for hyperopia on tear film and ocular surface. J Refract Surg 2002;18:113-23.

22 Siganos DS, Popescu CN, Siganos CS, et al. Tear secretion following excimer laser in situ keratomileusis. J Refract Surg 2002;18:124-6.

23 Wilson SE. Laser in situ keratomileusis-induced (presumed) neurotrophic epitheliopathy. Ophthalmology 2001;108:1082-7.

24 Sugar A, Rapuano CJ, Culbertson WW, et al. Laser in situ keratomileusis for myopia and astigmatism: safety and efficacy: a report by the American Academy of Ophthalmology. Ophthalmology 2002;109:175-87.

25 Breil P, Frisch L, Dick HB. Diagnosis and therapy of LASIK-induced neurotrophic epitheliopathy. Ophthalmologe 2002;99:53-7.

26 Moilanen JA, Vesaluoma MH, Muller $\sqcup$, et al. Long-term corneal morphology after PRK by in vivo confocal microscopy. Invest Ophthalmol Vis Sci 2003;44: 1064-9

27 Lee BH, McLaren J, Erie J, et al. Reinnervation in the cornea after LASIK. Invest Ophthalmol Vis Sci 2002;43:3660-4. 